\title{
Necessary steps in designing energy materials: from experiments to predictive modeling
}

\author{
Nitin Chopra \\ Editor-in-chief, Nanomaterials and Energy \\ Associate Professor, Metallurgical and Materials Engineering, The \\ University of Alabama, Tuscaloosa, AL, USA \\ Adjunct Professor, Department of Biological Sciences, The University of \\ Alabama, Tuscaloosa, AL, USA
}

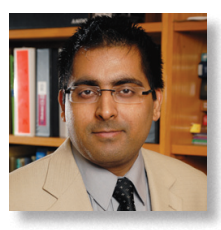

Experimental research in energy materials can be highly successful when fueled by predictive modeling and theoretical studies, where the latter have a strong potential for developing ideal materials and devices for novel alternative energy routes. Predictive modeling can include theoretical methods for material structural analysis and chemical processes or multiscale analysis for bridging various scales of material fundamentals. This issue of Nanomaterials and Energy focuses on a comprehensive review of branched nanostructures for electrochemical water splitting, understanding the strength of bonding in water molecules, and predictive modeling of a polycrystalline system.

The feature article by $\mathrm{Mao}^{1}$ presents a comprehensive review of threedimensional nanoarchitectured materials for solar electrochemical water splitting. This article highlights the importance of nanoscale heterostructuring for achieving modulated bandgap energies in materials and plasmonic activity, which are impossible to observe with single-component nanostructures. The tabulated data in terms of conditions and properties of such nanoscale heterostructures for water splitting are a unique compilation within this article. This comprehensive review also provides critical information about the growth, material selection, and water-splitting mechanisms that have been recently reported in the literature. The second research article, by Lamsal et al., ${ }^{2}$ focuses on predictive modeling and theory behind the equilibrium configuration of water molecules, a necessary fundamental aspect before we design materials for water splitting. This research paper describes ground-state energy and equilibrium geometry of water molecules. The approach is further extended to water-like molecules. Finally, the third research article by $\mathrm{Li}$ and Holland $^{3}$ demonstrates predictive modeling (based on effective medium approximation) for polycrystalline materials and their grain boundary network structure. As we aim to develop cheaper energy systems, it becomes critical that studies such as this are pursued, where theoretical modeling can be applied to understand the grain boundary diffusivity, crystal perfection, and texture.

With this issue of Nanomaterials and Energy, I welcome authors and readers to consider submitting articles in the area of predictive modeling and computational materials science, through which a significant impact can be made on making materials with right energy, surface, and electronic structure. These three aspects are fundamental to any alternative energy technology. This issue also assures our readers and authors that Nanomaterials and Energy is a highly focused platform for sharing novel results and ideas in the alternative energy sector. The selection of articles in every issue of Nanomaterials and Energy is done meticulously to meet the goals of the energy research area. Additionally, I welcome the readers to provide us feedback and allow us to better serve the science and technology of energy materials.

\section{REFERENCES}

1. Mao, Y. Branched nanostructures for photoelectrochemical water splitting. Nanomaterials and Energy 2014, 3 (4), 103-128.

2. Lamsal, C.; Mishra, M. R.; Ravindra, N. M. Equilibrium configuration of $\left(\mathrm{H}_{2} \mathrm{O}\right)_{n}$, for $n=1-3$. Nanomaterials and Energy 2014, 3 (4), 129-138.

3. Li, L.; Holland, S. Grain boundary diffusion: nonrandom topology and effective medium approximation. Nanomaterials and Energy 2014, 3 (4), 139-147. 